\title{
Approximation algorithms and hardness for domination with propagation
}

\author{
Ashkan Aazami \\ aaazami@uwaterloo.ca \\ Department of Combinatorics and Optimization \\ University of Waterloo
}

\author{
Michael David Stilp \\ mstilp3@gatech.edu \\ School of Industrial and Systems Engineering \\ Georgia Institute of Technology
}

November 2, 2018

\begin{abstract}
The power dominating set (PDS) problem is the following extension of the well-known dominating set problem: find a smallest-size set of nodes $S$ that power dominates all the nodes, where a node $v$ is power dominated if (1) $v$ is in $S$ or $v$ has a neighbor in $S$, or (2) $v$ has a neighbor $w$ such that $w$ and all of its neighbors except $v$ are power dominated. We show a hardness of approximation threshold of $2^{\log ^{1-\epsilon} n}$ in contrast to the logarithmic hardness for the dominating set problem. We give an $O(\sqrt{n})$ approximation algorithm for planar graphs, and show that our methods cannot improve on this approximation guarantee. Finally, we initiate the study of PDS on directed graphs, and show the same hardness threshold of $2^{\log ^{1-\epsilon} n}$ for directed acyclic graphs. Also we show that the directed PDS problem can be solved optimally in linear time if the underlying undirected graph has bounded tree-width.
\end{abstract}

Keywords: Approximation algorithms, Hardness of approximation, Dominating set, Power dominating set, Tree-width, Planar graphs, Greedy algorithms, PMU placement problem.

AMS subject classifications: $68 \mathrm{~W} 25 ; 90 \mathrm{C} 27$

\section{Introduction}

A dominating set of an (undirected) graph $G=(V, E)$ is a set of nodes $S$ such that every node in the graph is in $S$ or has a neighbor in $S$. The problem of finding a dominating set of minimum size is an important problem that has been extensively studied, especially in the last 20 years, see the books by Haynes et al. [17, 18. The problem is NP-hard [14, a simple greedy algorithm achieves a logarithmic approximation guarante $*$ [20], and, modulo the $\mathrm{P} \neq \mathrm{NP}$ conjecture, no polynomial time algorithm gives a better approximation guarantee [27, 13].

Our focus is on an extension called the Power Dominating Set (abbreviated as PDS) problem. Power domination is defined by two rules; the first rule is the same as the rule for the Dominating SET problem, but the second rule allows a type of indirect propagation. More precisely, given a set of nodes $S$, the set of nodes that are power dominated by $S$, denoted $\mathcal{P}_{S}$, is obtained as follows.

\footnotetext{
*An approximation algorithm for a (minimization) optimization problem means an algorithm that runs in polynomial time and computes a solution whose cost is within a guaranteed factor of the optimal cost; the approximation guarantee is the worst-case ratio, over all inputs of a given size, of the cost of the solution computed by the algorithm to the optimal cost.
} 
(Rule 1) if node $v$ is in $S$, then $v$ and all of its neighbors are in $\mathcal{P}_{S}$;

(Rule 2) (propagation) if node $v$ is in $\mathcal{P}_{S}$, one of its neighbors $w$ is not in $\mathcal{P}_{S}$, and all other neighbors of $v$ are in $\mathcal{P}_{S}$, then $w$ is inserted into $\mathcal{P}_{S}$.

The set $\mathcal{P}_{S}$ is independent of the sequence in which nodes are inserted by Rule 2. Otherwise, there is a minimal counter example with two maximal sequences of insertions and an "earliest" node that occurs in one sequence but not the other; this is not possible. The PDS problem is to find a node-set $S$ of minimum size that power dominates all nodes (i.e., find $S \subseteq V$ with $|S|$ minimum such that $\left.\mathcal{P}_{S}=V\right)$. We use $\operatorname{Opt}(G)$ to denote the size of an optimal solution for the PDS problem for a graph $G$. Throughout, we use $n$ to denote the number of nodes in the input graph.

For example, consider the planar graph in Figure 1: the graph has $t$ disjoint triangles, and three (mutually disjoint) paths such that each path has exactly one node from each triangle; note that $|V|=3 t$. The minimum dominating set has size $\Theta(|V|)$, since the maximum degree is 4 . The minimum power dominating set has size one - if $S$ has any one node of the innermost (first) triangle (like $v$ ), then $\mathcal{P}_{S}=V$.

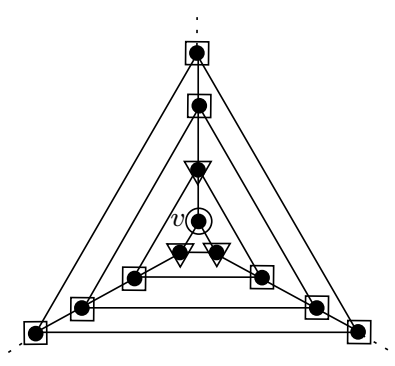

Figure 1: Illustrating those nodes power dominated by Rule 1 (denoted by a triangle) and Rule 2 (denoted by a square); the picked node is shown by a circle.

The PDS problem arose in the context of electric power networks, where the aim is to monitor all of the network by placing a minimum-size set of very expensive devices called phase measurement units; these units have the capability of monitoring remote elements via propagation (as in Rule 2); see Brueni [6], Baldwin et al. [4, and Mili et al. [28]. In the engineering literature, the problem is called the PMU placement problem.

Our motivation comes from the area of approximation algorithms and hardness results. The DominATing SET problem is a so-called covering problem; we wish to cover all nodes of the graph by choosing as few node neighborhoods as possible. In fact, the Dominating SET problem is a special case of the well-known Set Coverind problem.

Such covering problems have been extensively investigated. One of the key positive results dates from the 1970's, when Johnson [20, Lovász [26] and later Chvátal [8] showed that the greedy method achieves an approximation guarantee of $O(\log |V|)$ where $|V|$ denotes the size of the ground set, see also [30]. Several negative results (on the hardness of approximation) have been discovered over

\footnotetext{
${ }^{\dagger}$ In more detail, we apply Rule 1 to see that all the nodes of the innermost (first) triangle and one node of the second triangle are in $\mathcal{P}_{S}$; then by two applications of Rule 2 (to each of the nodes in the first triangle not in $S$ ), we see that the other two nodes of the second triangle are in $\mathcal{P}_{S}$; then by three applications of Rule 2 (to each of the nodes in the second triangle) we see that all three nodes of the third triangle are in $\mathcal{P}_{S}$; etc.

${ }^{\ddagger}$ Given a family of sets on a groundset, find the minimum number of sets whose union equals the groundset.
} 
the last few years: Lund and Yannakakis [27] showed that the SET CoverIng problem is hard to approximate within a ratio of $\Omega(\log n)$ and later, Feige [13] showed that it is hard to approximate within a ratio of $(1-\epsilon) \ln n$, modulo some variants of the $\mathrm{P} \neq \mathrm{NP}$ assumption.

A natural question is what happens to covering problems (in the setting of approximation algorithms and hardness results) when we augment the covering rule with a propagation rule. PDS seems to be a key problem of this type, since it is obtained from the Dominating SET problem by adding a simple propagation rule.

\subsection{Previous literature}

Apparently, the earliest publications on PDS are Brueni [6], Baldwin et al. [4, and Mili et al. [28]. Later, Haynes et al. [16] showed that the problem is NP-complete even when the input graph is bipartite; they presented a linear-time algorithm to solve PDS optimally on trees. Kneis et al. 23. generalized this result to a linear-time algorithm that finds an optimal solution for graphs that have bounded tree-width, relying on earlier results of Courcelle et al. [9]. Kneis et al. [23] also showed that PDS is a generalization of the Dominating Set problem as follows. Given a graph $G$ we can construct an augmented graph $G^{\prime}$ such that $S$ is an optimal solution for the Dominating SET problem on $G$ if and only if it is an optimal solution for PDS on $G^{\prime}$; the graph $G^{\prime}$ is obtained from $G$ by adding a new node $v^{\prime}$ for each node $v$ in $G$ and adding the edge $v v^{\prime}$. Guo et al. [15] developed a combinatorial algorithm based on dynamic-programming for optimally solving PDS on graphs of tree-width $k$. The running time of their algorithm is $O\left(c^{k^{2}} \cdot n\right)$ where $c$ is a constant. Guo et al. also compared the tractability of the Dominating SET problem versus PDS on several classes of graphs, that is, they study whether there are classes of graphs where the former problem is in $\mathrm{P}$ but the latter one is NP-hard; but they have no result that "separates" the two problems. Even for planar graphs, the Dominating SET problem is NP-hard [14], and the same holds for PDS [15]. Liao and Lee [25] proved that PDS on split graphs is NP-complete, and also they presented a polynomial time algorithm for solving PDS optimally on interval graphs. Dorfling and Henning computed the power domination number, i.e. the size of optimal power dominating set, for $n \times m$ grids [12. Brueni and Heath [7] have more results on PDS, especially the NP-completeness of PDS on planar bipartite graphs. To the best of our knowledge, no further results are known on solving the PDS problem, either optimally or approximately. Some of the results in this paper have appeared in the thesis of the second author [31], and in the proceedings of a workshop [1].

\subsection{Our contributions}

Our results substantially improve on the understanding of PDS in the context of approximation algorithms. In particular, we show a substantial gap between the approximation guarantees for the Dominating SET problem and PDS modulo a variant of the $\mathrm{P} \neq$ NP conjecture. This seems to be the first known "separation" result between the two problems, in any class of graphs.

- We present a reduction from the MinReP problem to the PDS problem that shows that PDS cannot be approximated within a factor of $2^{\log ^{1-\epsilon} n}$, unless NP $\subseteq \operatorname{DTIME}\left(n^{\text {polylog(n) }}\right)$.

- For undirected graphs, we introduce the notion of strong regions and weak regions as a means of obtaining lower bounds on the size of an optimal solution for PDS. Based on this, we develop an approximation algorithm for PDS that gives an approximation guarantee of $O(k)$ for graphs that have tree-width $k$. The algorithm requires the tree decomposition as 
part of the input, and runs in time $O\left(n^{3}\right)$ (independent of $k$ ). By slightly modifying this algorithm we get an algorithm that solves PDS optimally on trees. Our algorithm provides an $O(\sqrt{n})$-approximation algorithm for PDS on planar graphs because a tree decomposition of a planar graph with width $O(\sqrt{n})$ can be computed efficiently [2]. Moreover, we show that our methods (specifically, the lower bounds used in our analysis) cannot improve on our $O(\sqrt{n})$ approximation guarantee.

- We extend PDS in a natural way to directed graphs and prove that even for directed acyclic graphs, PDS is hard to approximate within the same threshold as for undirected graphs modulo the same complexity assumption.

- We give a linear-time algorithm based on dynamic-programming for DIRECTED PDS when the underlying undirected graph has bounded tree-width. This builds on results and methods of Guo et al. [15.

\section{$2 \quad$ PDS in Undirected Graphs}

In this section we prove a result on the hardness of approximating PDS by a reduction from the MinReP problem. In Section 2.1 we define the MinREP problem, and then we give a gap preserving reduction from MinREP to PDS in Section 2.2.

\subsection{The MinRep problem}

In the MinReP 24] problem we are given a bipartite graph $G=(A, B, E)$ with a partition of $A$ and $B$ into equal-sized subsets. Let $q_{A}$ and $q_{B}$ denote the number of sets in the partition of $A$ and $B$, respectively. Let $A=A_{1} \cup A_{2} \cup \cdots \cup A_{q_{A}}$ denote the partition of $A$, and let $B=B_{1} \cup B_{2} \cup \cdots \cup B_{q_{B}}$ denote the partition of $B$. This partition naturally defines a super bipartite graph $\mathcal{H}=(\mathcal{A}, \mathcal{B}, \mathcal{E})$. The super nodes of $\mathcal{H}$ are $A_{1}, A_{2}, \ldots, A_{q_{A}}$ and $B_{1}, B_{2}, \ldots, B_{q_{B}}$. There is a super edge between super nodes $A_{i}$ and $B_{j}$ if there exists some $a \in A_{i}$ and $b \in B_{j}$ such that $a b$ is an edge in $G$. We say that super edge $A_{i} B_{j}$ is covered by nodes $a, b$ if $a \in A_{i}, b \in B_{j}$, and there is an edge between $a$ and $b$ in $G$. Given $S \subseteq A \cup B$ we say that the super edge $A_{i} B_{j}$ is covered by $S$ if there exists $a, b \in S$ that covers $A_{i} B_{j}$. The goal in the MinReP problem is to pick a minimum-size set of nodes, $A^{\prime} \cup B^{\prime} \subseteq V(G)$, to cover all the super edges in $\mathcal{H}$. Note that we need a pair of nodes to cover a super edge, and the pair should induce an edge between the two super nodes of the super edge; moreover, a node in $A^{\prime} \cup B^{\prime}$ may be useful for covering more than one super edge. The following Theorem is from [24].

Theorem 2.1 (Theorem 5.4 in [24]) The MINREP problem cannot be approximated within ratio $2^{\log ^{1-\epsilon} n}$, for any fixed $\epsilon>0$, unless $N P \subseteq D T I M E\left(n^{\text {polylog }(n)}\right)$.

\subsection{The reduction to $\mathrm{PDS}$}

Theorem 2.2 The PDS problem cannot be approximated within ratio $2^{\log ^{1-\epsilon} n}$, for any fixed $\epsilon>0$, unless $N P \subseteq D T I M E\left(n^{\text {polylog }(n)}\right)$.

The reduction: Theorem 2.2 is proved by a reduction from the MinReP problem. We create an instance $\bar{G}=(\bar{V}, \bar{E})$ of the PDS problem from a given instance $G=(A, B, E)(\mathcal{H}=(\mathcal{A}, \mathcal{B}, \mathcal{E}))$ of the MinReP problem. The idea is to replace each super edge with a "cover testing gadget". 
1. Start with a copy of each node in $A \cup B$ in $\bar{G}$. For convenience, we use the same notation for nodes (and set of nodes) in $G$ and their copies in $\bar{G}$.

2. Add a new node $w^{*}$ to the graph $\bar{G}$, and connect $w^{*}$ to all nodes in $A \cup B$. Also add new nodes $w_{1}^{*}, w_{2}^{*}, w_{3}^{*}$ and connect them to $w^{*}$ (the nodes $w_{1}^{*}, w_{2}^{*}, w_{3}^{*}$ are added to force $w^{*}$ to be in any optimal solution. See the proof of Lemma 2.3 for more details).

3. $\forall i \in\left\{1, \ldots, q_{A}\right\}, j \in\left\{1, \ldots, q_{B}\right\}$ if $A_{i} B_{j}$ is a super edge, then do the following:

(a) Let $E_{i j}$ denote the set of edges between $A_{i}$ and $B_{j}$ in $G$ and let $\ell_{i j}$ denote $\left|E_{i j}\right|$ (see Figure $3(\mathrm{a})$ for an example $E_{11}$ has 3 edges, and $E_{12}$ has 4 edges). We denote the edges in $E_{i j}$ by $e_{1}, e_{2}, \cdots, e_{k}, \cdots$.

(b) Let $C_{i j}$ be a cycle of $3 \ell_{i j}$ nodes. We sequentially label the nodes of $C_{i j}$ as $u_{1}, v_{1}, w_{1}$, $u_{2}, v_{2}, w_{2}, \cdots, u_{k}, v_{k}, w_{k}, \cdots$ (informally speaking, we associate each triple $u_{k}, v_{k}, w_{k}$ with an edge $e_{k}$ of $\left.E_{i j}\right)$. Make $\lambda=4$ new copies of the graph $C_{i j}$ ( $\lambda$ can be any constant greater than 3 ; refer to the proof of Lemma 2.3 for more details). For each edge $e_{k}=a_{k} b_{k} \in E_{i j}$ and for each of the 4 copies of $C_{i j}$, we add an edge from $a_{k}$ to $u_{k}$ and an edge from $b_{k}$ to $v_{k}$. See Figures 2(a), 2(b) for an illustration.

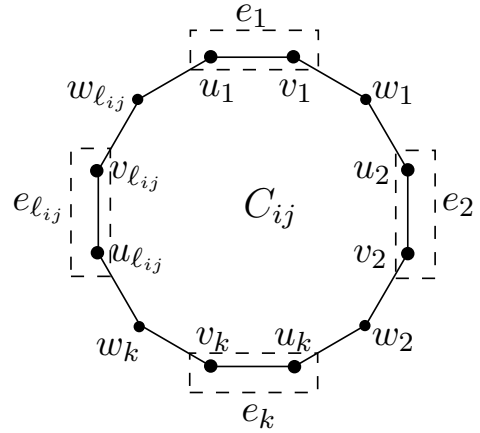

(a) The $C_{i j}$ graph

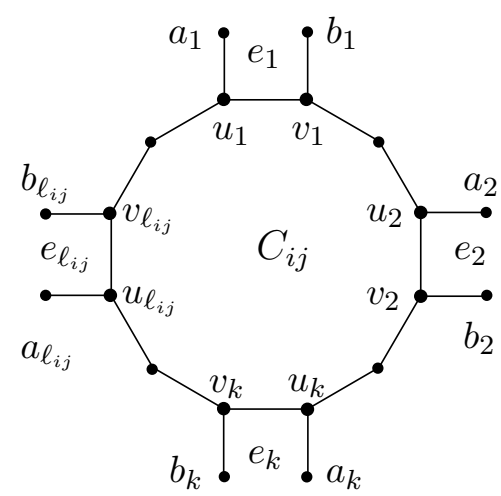

(b) Edges between $C_{i j}$ and $A_{i} \cup B_{j}$.

Figure 2: The cover testing gadget.

4. Let $\bar{G}=(\bar{V}, \bar{E})$ be the obtained graph (see Figure 3 for an illustration).

Let $S$ be a feasible solution for the resulting PDS instance $\bar{G}$, and suppose $w^{*} \in S$. Then all of the nodes in $A \cup B$ are power dominated (by Rule 1 of PDS). Now consider a gadget $C_{i j}$, and assume a node $v$ of $C_{i j}$ is in $S$. By applying Rule 1 once and then repeatedly applying Rule 2 of PDS, the gadget $C_{i j}$ will be completely power dominated, that is, all nodes of the gadget will be in $\mathcal{P}_{S}$.

The next lemma shows that the size of an optimal solution in PDS is exactly one more than the size of an optimal solution in MinReP. The number of nodes in the constructed graph is equal to $|V(\bar{G})|=4+|V(G)|+3 \lambda|E(G)|$. This will complete the proof of Theorem 2.2 by showing that the above reduction is a gap preserving reduction from MinREP to PDS with the same gap (hardness ratio) as the MinReP problem. 
Lemma 2.3 $A^{*} \cup B^{*}$ is an optimal solution to the instance $G=(A, B, E)$ of the MinREP problem if and only if $S^{*}=A^{*} \cup B^{*} \cup\left\{w^{*}\right\} \subseteq V(\bar{G})$ is an optimal solution to the instance $\bar{G}$ of the PDS problem.

Proof: First, we claim that $w^{*}$ should be in any optimal solution of the PDS instance $\bar{G}$. Suppose that $w^{*}$ is not in some optimal solutions. Then, in order to power dominate the nodes $w^{*}, w_{1}^{*}, w_{2}^{*}, w_{3}^{*}$ in $\bar{G}$, the set $S$ must contain at least two of the nodes (leaves) $w_{1}^{*}, w_{2}^{*}, w_{3}^{*}$. This is a contradiction, since we can replace these 2 nodes by $w^{*}$ and obtain a smaller feasible solution.

Assume that $A^{*} \cup B^{*}$ is a feasible solution for the MinRep instance $G$. We will show that $S=A^{*} \cup B^{*} \cup\left\{w^{*}\right\}$ is a feasible solution to the PDS instance $\bar{G}$. Note that all nodes in $A \cup B \cup$ $\left\{w^{*}, w_{1}^{*}, w_{2}^{*}, w_{3}^{*}\right\}$ are power dominated by applying Rule 1 on $w^{*}$. Now, we only need to show that all nodes in the gadgets $C_{i j}$ are power dominated. Consider any super edge $A_{i} B_{j}$ of $\mathcal{H}$. The set $A^{*} \cup B^{*}$ covers all the super edges in $\mathcal{H}$. So there exists a pair of nodes $a_{k} \in A^{*} \cap A_{i}, b_{k} \in B^{*} \cap B_{j}$ that induces an edge of $G$. Since $a_{k}$ and $b_{k}$ are in $S$, their neighbors, $u_{k}$ and $v_{k}$, in each of the $\lambda=4$ copies of $C_{i j}$ in $\bar{G}$, will be power dominated by applying Rule 1 . Then the nodes $u_{k}$ and $v_{k}$ in each copy of $C_{i j}$ will power dominate the entire cycle by repeatedly applying Rule 2 . To see this, note that any node in $C_{i j}$ has exactly 2 neighbors in $C_{i j}$ and at most 1 neighbor not in $C_{i j}$. The neighbors not in $C_{i j}$ are from $A_{i} \cup B_{j}$, and they are power dominated by $w^{*}$. Hence, if a node in $C_{i j}$ and one of its neighbors in $C_{i j}$ are power dominated, then by applying Rule 2 the other neighbor in $C_{i j}$ will be power dominated. Hence, by starting from $v_{k}$ and repeatedly applying Rule 2, we can sequentially power dominate the nodes in $C_{i j}$. This shows that $S$ power dominates all nodes in $\bar{G}$. Therefore, $\operatorname{Opt}(\bar{G})$ is at most $\left|A^{*} \cup B^{*}\right|+1$.

Let $S^{*} \subseteq V(\bar{G})$ be an optimal solution for PDS. By the above claim, $w^{*}$ is in $S^{*}$. Now define $A^{\prime}=A \cap S^{*}$ and $B^{\prime}=B \cap S^{*}$. First we prove that any optimal solution of PDS is contained in $A \cup B \cup\left\{w^{*}\right\}$, and then we show that $A^{\prime} \cup B^{\prime}$ covers all super edges of the MinRep instance $G$. Suppose that $S^{*}$ contains some nodes not in $A \cup B \cup\left\{w^{*}\right\}$. Hence, there are some gadgets that are not completely power dominated by $S^{*} \cap\left(A \cup B \cup\left\{w^{*}\right\}\right)$. Let $C_{i j}$ be such a gadget. By symmetry each of the $\lambda=4$ copies of $C_{i j}$ is not completely power dominated. Therefore, the optimal solution $S^{*}$ needs to have at least 3 nodes from the 4 copies of $C_{i j}$. By removing these 3 nodes from $S^{*}$ and

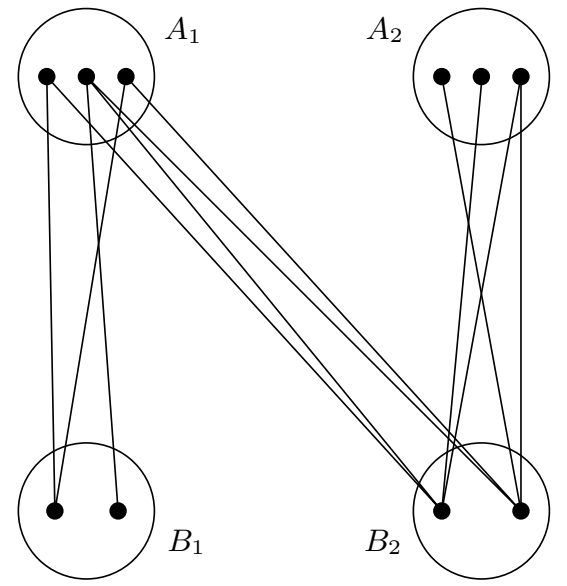

(a) MinRep Instance $G$

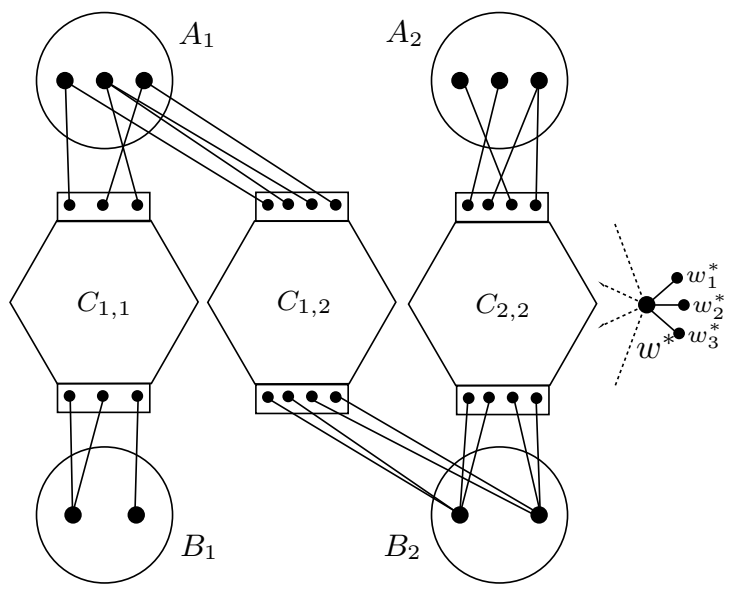

(b) PDS instance $\bar{G}$ : For each super edge $A_{i} B_{j}$ we show only 1 copy of $C_{i j}$; in fact $\bar{G}$ has $\lambda=4$ copies of $C_{i j}$.

Figure 3: The hardness construction 
adding $a_{k} \in A_{i}$ and $b_{k} \in B_{j}$ to $S^{*}$ for some arbitrary edge $a_{k} b_{k} \in E_{i j}$, we can power dominate all of the 4 copies of $C_{i j}$. This contradicts the minimality of $S^{*}$, and proves that $S^{*} \subseteq A \cup B \cup\left\{w^{*}\right\}$. To see that $A^{\prime} \cup B^{\prime}$ covers all super edges, note the following: suppose no node from any copy of $C_{i j}$ is in the optimal solution; then any $C_{i j}$ can be power dominated only by taking a pair of nodes $a \in A_{i}, b \in B_{j}$ that induces an edge of $G$. This completes the proof of the lemma.

\section{Approximation Algorithms for Planar Graphs}

In this section we describe an $O(k)$-approximation algorithm for PDS in graphs with tree-width $k$; the running time is $O\left(n^{3}\right)$, independent of $k$. This algorithm gives an $O(\sqrt{n})$-approximation algorithm for PDS in planar graphs, since the tree-width of a planar graph $G$ with $n$ nodes is $O(\sqrt{n})$ and in $O\left(n^{\frac{3}{2}}\right)$ time we can find an $O(\sqrt{n})$ tree-width decomposition of the given planar graph $G$ [2]. Finally, we show that the analysis of our algorithm is tight on planar graphs. We use PLANAR PDS to denote the special case of the PDS problem where the graph is planar.

Definition 3.1 [1] $A$ tree decomposition of a graph $G=(V, E)$ is a pair $\left\langle\left\{X_{i} \subseteq V \mid i \in I\right\}, T=\right.$ $(I, F)\rangle$ such that $T$ is a tree with $V(T)=I, E(T)=F$, and satisfying the following properties:

(T1) $\bigcup_{i \in I} X_{i}=V$, and every edge $u v \in E$ has both ends in some $X_{i}$,

(T2) For all $i, j, k \in I$ if $j$ is on the unique path from $i$ to $k$ in $T$ then we have: $X_{i} \cap X_{k} \subseteq X_{j}$,

The width of $\left\langle\left\{X_{i} \mid i \in I\right\}, T\right\rangle$ is the $\max _{i \in I}\left|X_{i}\right|-1$. The tree-width of $G$ is defined as the minimum width over all tree decompositions. The nodes of the tree are called T-nodes and the sets $X_{i}$ are called bags.

A nice tree decomposition is a tree decomposition $\left\langle\left\{X_{i} \subseteq V \mid i \in I\right\}, T=(I, F)\right\rangle$, where $T$ is a rooted tree in which each node has at most 2 children. If a node $i \in I$ has two children $j, k$ then $X_{i}=X_{j}=X_{k}$ ( $i$ is called a JoIn node), and if $i$ has one child $j$ then either $X_{j} \subset X_{i}$ and $\left|X_{i} \backslash X_{j}\right|=1$ or $X_{i} \subset X_{j}$ and $\left|X_{j} \backslash X_{i}\right|=1$ ( $i$ is called an InsERT or a Forget node, respectively).

We introduce the notion of a strong region before presenting our algorithm. Informally speaking, a set of nodes $R \subseteq V$ is called strong if every feasible solution to the PDS problem has a node of $R$. For a graph $G=(V, E)$, the neighborhood of $R \subseteq V$ is $n b r(R)=\{v \in V \mid \exists u v \in E, u \in R, v \notin R\}$, and the exterior of $R$ is defined by $\operatorname{ext}(R)=n b r(V \backslash R)$, i.e., $\operatorname{ext}(R)$ consists of the nodes in $R$ that are adjacent to a node in $V \backslash R$.

Definition 3.2 Given a graph $G=(V, E)$ and a set $S \subseteq V$, the subset $R \subseteq V$ is called an $S$-strong region if $R \nsubseteq \mathcal{P}_{S \cup n b r(R)}$, otherwise, the set $R$ is called an $S$-weak region. The region $R$ is called minimal $S$-strong if it is an $S$-strong region and $\forall r \in R, R-r$ is an $S$-weak region.

It is easy to check from the definition that an $S$-strong region is also an $\emptyset$-strong (or shortly strong) region. Any feasible solution to the PDS problem needs to have at least one node from every strong region.

Lemma 3.3 A subset $R \subseteq V$ is an $S$-strong region if and only if for every feasible solution $S \cup S^{*}$ of $G$, we have $R \cap\left(S^{*} \backslash S\right) \neq \emptyset$. 
Proof: It can be seen that the set $S \cup(V \backslash R)$ will power dominate the same set of nodes in $R$ that can be power dominated by $S \cup n b r(R)$; this is valid for any subset $R \subseteq V$.

Let $R$ be an $S$-strong region. By the definition of a strong region we have $R \nsubseteq \mathcal{P}_{S \cup n b r(R)}$. Hence, by the above claim $R \nsubseteq \mathcal{P}_{S \cup(V \backslash R)}$. This shows that every feasible solution $S^{*}$ needs to have at least one node from $R$ that is not in $S$.

Now assume that for every feasible solution $S^{*}$ of $G$ we have $R \cap\left(S^{*} \backslash S\right) \neq \emptyset$. Suppose that $R$ is an $S$-weak region, so by the definition of a weak region we have $R \subseteq \mathcal{P}_{S \cup n b r(R)}$. It follows that $S^{*}=S \cup(V \backslash R)$ is a feasible solution, but $R$ has no intersection with $S^{*} \backslash S \subseteq(V \backslash R)$. This is a contradiction, so $R$ is an $S$-strong region.

Our algorithm makes one level-by-level and bottom-to-top pass over the tree $T$ of the tree decomposition of $G$ and constructs a solution $S$ for PDS (initially, $S=\emptyset$ ). At each node $r_{j}$ of $T$ we check whether the union of the bags in the subtree rooted at $r_{j}$ forms an $S$-strong region; if yes, then the bag $X_{r_{j}}$ of $r_{j}$ is added to $S$, otherwise $S$ is not updated. The key point in the analysis is to show that $\operatorname{Opt}(G) \geq m$, where $m$ is the number of nodes of $T$ where we updated $S$.

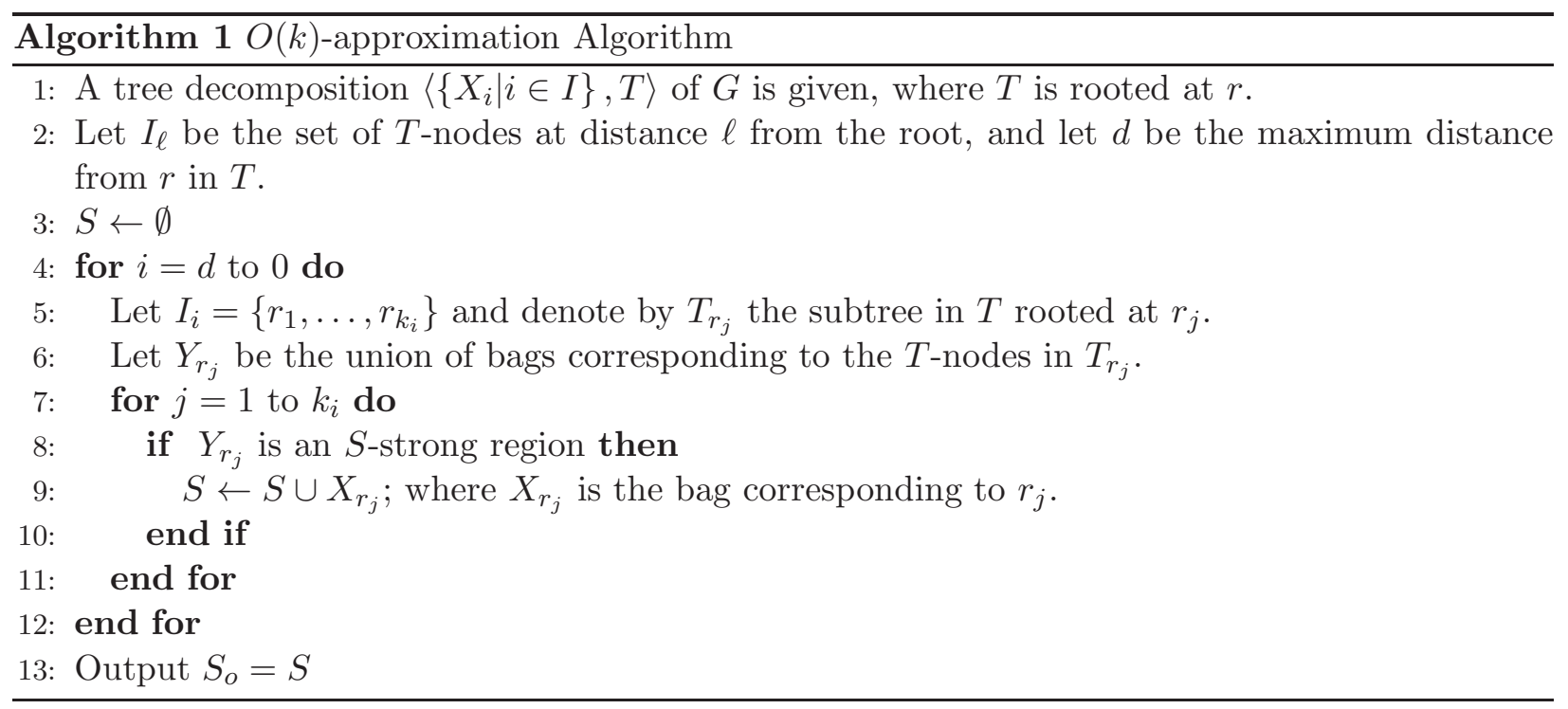

\subsection{Analysis of the algorithm}

In this subsection we show that our algorithm has an approximation guarantee of $O(k)$. Let $G=(V, E)$ denote the input graph, and let $S \subseteq V$ be any set of nodes.

Lemma 3.4 Suppose $Z$ is an $S$-weak region such that $\operatorname{ext}(Z) \subseteq S$. Then we have $Z \subseteq \mathcal{P}_{S}$.

Proof: Let $Y=\operatorname{ext}(Z)$, it is easy to check that $n b r(Z \backslash Y) \subseteq \operatorname{ext}(Z)$. We claim that $Z \backslash Y$ is an $S$-weak region. Let $S^{*}=V \backslash(Z \cup S)$, it is easy to check that $S \cup S^{*}$ is a feasible solution for the graph $G$, but $S^{*} \cap(Z \backslash Y)=\emptyset$. Hence, by Lemma 3.3, $Z \backslash Y$ is not an $S$-strong region, and so it is an $S$-weak region. Thus $Z \backslash Y \subseteq \mathcal{P}_{S \cup n b r(Z \backslash Y)} \subseteq \mathcal{P}_{S \cup \operatorname{ext}(Z)}=\mathcal{P}_{S}$ and this implies that $Z \subseteq \mathcal{P}_{S}$ as $Y=\operatorname{ext}(Z) \subseteq S$. 
Lemma 3.5 Let $Z \subseteq V$ be an $S$-strong region. Suppose that $Y$ is a subset of $V$ such that $Y \subseteq \mathcal{P}_{S}$ and $\operatorname{ext}(Y) \subseteq S$. Then $Z \backslash Y$ is an $S$-strong region.

Proof: Assume for the sake of contradiction that $Z \backslash Y$ is an $S$-weak region. Then by the definition of strong regions we have: $Z \backslash Y \subseteq \mathcal{P}_{S \cup n b r(Z \backslash Y)}$. It is easy to see that $n b r(Z \backslash Y) \subseteq n b r(Z) \cup \operatorname{ext}(Y)$. This implies that $Z \backslash Y \subseteq \mathcal{P}_{S \cup n b r(Z \backslash Y)} \subseteq \mathcal{P}_{S \cup n b r(Z) \cup \operatorname{ext}(Y)}=\mathcal{P}_{S \cup n b r(Z)}$. The condition in the lemma states that $Y \subseteq \mathcal{P}_{S} \subseteq \mathcal{P}_{S \cup n b r(Z)}$. Hence, we get $Z=(Z \backslash Y) \cup(Z \cap Y) \subseteq \mathcal{P}_{S \cup n b r(Z)}$, which means that $Z$ is an $S$-weak region. This is a contradiction, so the lemma is proved.

Theorem 3.6 Given a graph $G=(V, E)$ and a tree decomposition of $G$ of width $k$ as input, Algorithm 1 runs in time $O(n \cdot|E|)$, and achieves an approximation guarantee of $(k+1)$.

Proof: First, we show that the solution $S_{o}$ found by the algorithm is feasible. Then we prove the approximation guarantee, and establish the running time.

For any node $q$ of $T$, recall that $Y_{q}$ denotes the union of the bags corresponding to the $T$-nodes in the subtree rooted at $q$ in $T$; let $G_{q}$ denote the subgraph of $G$ induced by $Y_{q}$. We claim that $\operatorname{ext}\left(Y_{q}\right) \subseteq X_{q}$. Suppose that $q$ has $m$ children in $T$, call them $c_{1}, \ldots, c_{m}$. For each edge $q c_{j}$ $(j=1, \cdots, m)$, the set $X_{q} \cap X_{c_{j}}$ separates $Y_{c_{j}}$ from the rest of the graph, that is, every path between a node in $Y_{c_{j}}$ and a node in $V \backslash Y_{c_{j}}$ contains a node of $X_{q} \cap X_{c_{j}}$ (see Lemma 12.3.1 in [11]). Thus, $\operatorname{ext}\left(Y_{c_{j}}\right) \subseteq X_{q} \cap X_{c_{j}} \subseteq X_{q}$, and hence, for $Y_{q}=X_{q} \cup Y_{c_{1}} \cup \cdots \cup Y_{c_{m}}$, we have $\operatorname{ext}\left(Y_{q}\right) \subseteq X_{q}$.

We use induction on the height of the subtree of $T$ rooted at $q$ to prove the following: if $Y_{q}$ is $S^{*}$-strong, then $Y_{q} \subseteq \mathcal{P}_{S^{*} \cup X_{q}}$, where $S^{*}$ denotes the solution just before the algorithm examines $Y_{q}$. The statement clearly holds when $q$ is a leaf of $T$ (since $Y_{q}=X_{q}$ ). Otherwise, let $c_{1}, \ldots, c_{m}$ be the children of $q$ in $T$. For each $j=1, \ldots, m$, when the algorithm examined $Y_{c_{j}}$, either $Y_{c_{j}}$ was $S$-weak, in which case (by Lemma 3.4) we have $Y_{c_{j}} \subseteq \mathcal{P}_{S \cup \operatorname{ext}\left(Y_{c_{j}}\right)} \subseteq \mathcal{P}_{S \cup\left(X_{c_{j}} \cap X_{q}\right)} \subseteq \mathcal{P}_{S^{*} \cup X_{q}}$ or $Y_{c_{j}}$ was $S$-strong in which case $Y_{c_{j}} \subseteq \mathcal{P}_{S \cup X_{c_{j}}}$ by induction (note that $S \cup X_{c_{j}} \subseteq S^{*}$ ); we use $S$ to denote the solution just before the algorithm examines $Y_{c_{j}}$. Hence, $Y_{q}=Y_{c_{1}} \cup \cdots \cup Y_{c_{m}} \cup X_{q} \subseteq \mathcal{P}_{S^{*} \cup X_{q}}$.

The above statement implies that $V \subseteq \mathcal{P}_{S_{o}}$ because at the step when the algorithm examines the root $r$ of $T$ either

(i) $Y_{r}$ is $S$-strong, so $S_{o}=S \cup X_{r}$, and $Y_{r} \subseteq \mathcal{P}_{S \cup X_{r}}=\mathcal{P}_{S_{o}}$; or

(ii) $Y_{r}$ is $S$-weak, and $Y_{r} \subseteq \mathcal{P}_{S \cup \operatorname{ext}\left(Y_{r}\right)}=\mathcal{P}_{S_{o}}$; since $Y_{r}=V(G)$ and $\operatorname{ext}\left(Y_{r}\right)=\emptyset$.

To show that the approximation guarantee is $(k+1)$ we will construct a set $\Delta$ of pairwise disjoint strong regions $R_{1}, R_{2}, \ldots$, such that there is a strong region $R_{j}$ corresponding to each step of the algorithm that adds a non empty bag $X_{q_{j}}$ to $S$. Thus $\left|S_{o}\right| \leq(k+1)|\Delta|$ since each bag has $\leq k+1$ nodes, and $\operatorname{Opt}(G) \geq|\Delta|$ because every feasible solution has size $\geq|\Delta|$, by Lemma 3.3. Hence, $\left|S_{o}\right| \leq(k+1) \operatorname{Opt}(G)$. We construct the sets $R_{1}, R_{2}, \ldots$, during the execution of the algorithm as follows. Suppose the algorithm finds $Y_{q}$ to be $S$-strong while examining a node $q$ of $T$. Let $q_{1}, \ldots, q_{\ell-1}$ be the nodes of $T$ where the algorithm updated the solution before examining $q$, and let $S$ be the solution just before the algorithm examines $q$. Then define $R_{\ell}=Y_{q_{\ell}} \backslash\left(Y_{q_{1}} \cup \cdots \cup Y_{q_{\ell-1}}\right)$, where $q_{\ell}=q$. We claim that $R_{\ell}$ is an $S$-strong region. For each strong region $Y_{q_{j}}(j=1, \ldots, \ell-1)$ we have seen that $\operatorname{ext}\left(Y_{q_{j}}\right) \subseteq X_{q_{j}} \subseteq S$ and $Y_{q_{j}} \subseteq \mathcal{P}_{S}$; note that the algorithm added $X_{q_{j}}$ to the solution since $Y_{q_{j}}$ was a strong region. It follows that $\operatorname{ext}\left(Y_{q_{1}} \cup \cdots \cup Y_{q_{\ell-1}}\right) \subseteq S$, and $Y_{q_{1}} \cup \cdots \cup Y_{q_{\ell-1}} \subseteq \mathcal{P}_{S}$. Hence, by Lemma 3.5, the set $R_{\ell}$ is an $S$-strong region. Clearly, the sets $R_{1}, R_{2}, \ldots$, are pairwise disjoint. This completes the construction of $\Delta$. 
Consider the running time. Without loss of generality we can assume that the given tree decomposition of width $k$ has at most $4 n$ bags (see Lemma 13.1.2 in 22]). Using standard algorithmic techniques we can test in $O(|E|)$ time whether a given set $R \subseteq V$ is an $S$-strong region (we compute $\mathcal{P}_{S \cup n b r(R)}$ and check if it contains $R$ ). Therefore, our algorithm has a running time of $O(n \cdot|E|)$.

It is known that planar graphs have tree-width $O(\sqrt{n})$, and such a tree decomposition can be found in $O\left(n^{\frac{3}{2}}\right)$ time [2]. This fact together with the above theorem proves the following theorem.

Theorem 3.7 Algorithm 1 achieves an approximation guarantee of $O(\sqrt{n})$ for the PLANAR PDS problem.

As mentioned earlier, Haynes et al. 16] presented a linear-time algorithm for optimally solving PDS on trees. By modifying Algorithm 1, we can solve PDS optimally on trees. The resulting algorithm differs from the algorithm of Haynes et al. since our algorithm uses strong regions. Informally, the algorithm makes a level-by-level and bottom-to-top pass over the tree $G$. At a node $v$ of the tree $G$ if the set of nodes in the subtree rooted at $v$ forms a strong region, then we add $v$ to the solution, otherwise we skip $v$. Formally, we define $X_{v}=\{v\}$ for each $v \in V(G)$, and we run Algorithm 1 on the tree $G$. Note that defining bags in this way does not give a tree decomposition of $G$.

Theorem 3.8 A modification of Algorithm 1 runs in time $O(n \cdot|E|)$ and solves PDS optimally on trees.

\subsection{Lower bounds via disjoint strong regions}

In this part we show that any approximation algorithm for PDS that uses the number of disjoint strong regions as a lower bound has an approximation guarantee of $\Omega(\sqrt{n})$. In proposition 3.12 we give a lower bound on the optimal value for PDS on an $\ell \times m$ grid. Independently, [12] gave a stronger result for this.

Lemma 3.9 Any minimal $S$-strong region is connected.

Proof: Assume that $R$ is a minimal $S$-strong region that is not connected. Let $C \subset R$ be a connected component of $\mathrm{R}$. The set $C$ is an $S$-weak region since $R$ is a minimal $S$-strong region. By the definition of a weak region we have $C \subseteq \mathcal{P}_{S \cup n b r(C)}$. The set $C$ is a connected component of $R$, so the neighborhood of $C$ has no intersection with $R \backslash C$. This implies that $n b r(C) \subseteq n b r(R)$, and consequently we have $C \subseteq \mathcal{P}_{S \cup n b r(C)} \subseteq \mathcal{P}_{S \cup n b r(R)}$. The same argument as above shows that $R \backslash C \subseteq \mathcal{P}_{S \cup n b r(R)}$. Hence, $R \subseteq \mathcal{P}_{S \cup n b r(R)}$ which is a contradiction.

Lemma 3.10 The number of disjoint strong regions in an $\ell \times m$ grid is exactly one.

Proof: For the sake of contradiction, assume that the given grid has two disjoint strong regions. Take as few nodes as possible from these strong regions until we get minimal strong regions, say $R_{1}$ and $R_{2}$. It is easy to check that the set of nodes of any row or any column of the grid power dominates all nodes in the grid. By Lemma 3.3, $R_{1}$ and $R_{2}$ should have at least one node from every feasible solution. In the other words, $R_{1}$ and $R_{2}$ must have at least one node from each row and also from each column. By lemma 3.9, we know that $R_{1}$ and $R_{2}$ induce connected subgraphs. Hence, in $R_{1}$ there is a path from a node in the top row to a node in the bottom row, and also in $R_{2}$ 
there is a path from a node in the rightmost column to a node in the leftmost column. Obviously these two paths share a common node. This is a contradiction, since $R_{1}$ and $R_{2}$ are assumed to be disjoint.

We denote by $\mathcal{P}_{S}^{i}$ a set of nodes that are power dominated after applying propagation rule, Rule 2 , for $i$ number of times to $\mathcal{P}_{S}^{0}=S \cup n b r(S)$. Obviously this depends on the order of applying the propagation rule. We use the notation without specifying the order of applying the propagation rule.

Lemma 3.11 (Propagation lemma) Given an ordering of propagation rules applied to $S \cup n b r(S)$ with $\mathcal{P}_{S}^{k}=\mathcal{P}_{S}$ we have: $\left|\operatorname{ext}\left(\mathcal{P}_{S}^{j}\right)\right| \leq\left|\operatorname{ext}\left(\mathcal{P}_{S}^{i}\right)\right|, \forall 0 \leq i<j \leq k$.

Proof: We will prove that $\left|\operatorname{ext}\left(\mathcal{P}_{S}^{i+1}\right)\right| \leq\left|\operatorname{ext}\left(\mathcal{P}_{S}^{i}\right)\right|$, for all $0 \leq i \leq k-1$. Consider the set $\mathcal{P}_{S}^{i}$ and assume that in the $(i+1)$-st step we apply Rule 2 to $v \in \operatorname{ext}\left(\mathcal{P}_{S}^{i}\right)$ and power dominate $u$; i.e. $u \in \mathcal{P}_{S}^{i+1}, u \notin \mathcal{P}_{S}^{i}$. To apply Rule 2 to $v$ all neighbors of $v$ except $u$ should be power dominated, so we have $n b r(v) \backslash \mathcal{P}_{S}^{i}=\{u\}$. Also since we power dominate $u$ at step $(i+1)$, we have $n b r(v) \subseteq \mathcal{P}_{S}^{i+1}$. Therefore, $v$ is not in $\operatorname{ext}\left(\mathcal{P}_{S}^{i+1}\right)$, but $u$ may be in the exterior of $\mathcal{P}_{S}^{i+1}$. Hence, we have $\operatorname{ext}\left(\mathcal{P}_{S}^{i+1}\right) \subseteq$ $\left(\operatorname{ext}\left(\mathcal{P}_{S}^{i}\right) \backslash\{v\}\right) \cup\{u\}$. It follows that $\left|\operatorname{ext}\left(\mathcal{P}_{S}^{i+1}\right)\right| \leq\left|\operatorname{ext}\left(\mathcal{P}_{S}^{i}\right) \backslash\{v\}\right|+|\{u\}|=\left|\operatorname{ext}\left(\mathcal{P}_{S}^{i}\right)\right|$.

Proposition 3.12 Let $G$ be an $\ell \times m$ grid with $\ell \leq m$, then $\operatorname{Opt}(G)=\Theta(\ell)$.

Proof: First note that any row or any column of the grid power dominates all nodes. In the following we prove that any feasible solution of PDS needs to have at least $\frac{l-1}{5}$ nodes. Assume that there exists $S \subseteq V$ such that $|S|<\frac{l-1}{5}$ and $\mathcal{P}_{S}=V(G)$. The maximum degree in $G$ is 4 , so we have $\left|\operatorname{ext}\left(\mathcal{P}_{S}^{0}\right)\right| \leq|S \cup n b r(S)|<\frac{l-1}{5} \cdot 5=l-1$. Therefore, $\mathcal{P}_{S}^{0}$ contains no full row or no full column of $G$. The set $S$ power dominates $G$, so there is an $i$ such that $\mathcal{P}_{S}^{i}$ contains a full row or a full column. Consider the smallest $i$ with this property. Hence, $\mathcal{P}_{S}^{i-1}$ has no full row or full column. But some row or some column must have at least $l-1$ nodes in $\mathcal{P}_{S}^{i-1}$ since $\mathcal{P}_{S}^{i}$ contains an entire row or an entire column. Without loss of generality assume that these $l-1$ nodes are from a column. By the definition of $i$, each of the $l-1$ nodes in this column are in a row which is not a subset of $\mathcal{P}_{S}^{i-1}$. Therefore, there are at least $l-1$ rows with at least one node in $\mathcal{P}_{S}^{i-1}$ and at least one node not in $\mathcal{P}_{S}^{i-1}$. This implies that $\left|\operatorname{ext}\left(\mathcal{P}_{S}^{i-1}\right)\right| \geq l-1$. Finally, by using Lemma 3.11 we get the following contradiction: $l-1>\left|\mathcal{P}_{S}^{0}\right| \geq\left|\operatorname{ext}\left(\mathcal{P}_{S}^{0}\right)\right| \geq\left|\operatorname{ext}\left(\mathcal{P}_{S}^{i-1}\right)\right| \geq l-1$.

Consider any approximation algorithm for PDS that uses only the number of disjoint strong regions as a lower bound on the size of an optimal solution. By Lemma 3.10, this algorithm finds a lower bound of 1 on the size of an optimal solution on a grid. The $\sqrt{n} \times \sqrt{n}$ grid has an optimal solution of size $\Theta(\sqrt{n})$ by Proposition 3.12. This shows that the approximation guarantee of the algorithm is $\Omega(\sqrt{n})$, even on planar graphs.

Proposition 3.13 Consider any approximation algorithm for PDS that uses only the number of disjoint strong regions as a lower bound on the optimal value. Then the approximation guarantee is $\Omega(\sqrt{n})$.

\section{PDS in Directed Graphs}

In this section we extend the PDS problem to directed graphs to obtain the Directed Power Dominating Set (DiRected PDS) problem. Our motivation for studying the directed problem comes from theoretical considerations. The Dominating SeT problem is studied on both 
undirected and directed graphs, and there is extensive literature on the latter (see [17, 18]). The similarities between the Dominating SET problem and the PDS problem led us to define and study the DiRECTED PDS problem. We give a result on the hardness of approximation of DiRECTED PDS. Then we reformulate the DIRECTED PDS problem in terms of valid coloring of the edges. Using this, we design an algorithm for solving DiRECTED PDS in linear-time on a special class of directed graphs.

Let $G=(V, E)$ be a directed graph. A node $w$ is called an out-neighbor (in-neighbor) of a node $v$ if there is a directed edge from $v$ to $w$ (from $w$ to $v$ ) in $G$. The number of out-neighbors (in-neighbors) of a node $v$ is called the out-degree (in-degree) of $v$ and is denoted by $d_{G}^{+}(v)$ (or similarly $\left.d_{G}^{-}(v)\right)$. For a set of nodes $X$, the subgraph of $G$ induced by $X$ is denoted by $G[X]$. The directed graphs that we consider here have no loops nor parallel edges, but may have two edges with different directions on the same two end nodes (we call such edges antiparallel). Given a directed graph $G$ by the underlying undirected graph we mean the undirected graph obtained from $G$, by removing the direction of edges and also removing any parallel edges that are introduced after removing the directions.

Definition 4.1 (the Directed PDS problem) Let $G$ be a directed graph. Given a set of nodes $S \subseteq V(G)$, the set of nodes that are power dominated by $S$, denoted by $\mathcal{P}_{S}$, is obtained as follows:

(D1) if node $v$ is in $S$, then $v$ and all of its out-neighbors are in $\mathcal{P}_{S}$;

(D2) (propagation) if node $v$ is in $\mathcal{P}_{S}$, one of its out-neighbors $w$ is not in $\mathcal{P}_{S}$, and all other out-neighbors of $v$ are in $\mathcal{P}_{S}$, then $w$ is inserted into $\mathcal{P}_{S}$.

We say that $S$ power dominates $G$ if $\mathcal{P}_{S}=V(G)$. The DiRECTED PDS problem is to find a node set $S$ with minimum size that power dominates all the nodes in $G$.

We prove a threshold of $2^{\log n^{1-\epsilon}}$ for the hardness of approximation of DiRECTED PDS modulo the same complexity assumption as in Theorem 2.2. The proof uses a reduction from the MinREP problem to the DiRECTED PDS problem in a directed acyclic graph. This reduction is similar to the reduction in Theorem 2.2, the main difference comes from the gadget for modeling the super edges.

Theorem 4.2 The DIRECTED PDS problem even when restricted to directed acyclic graphs cannot be approximated within ratio $2^{\log ^{1-\epsilon} n}$, for any fixed $\epsilon>0$, unless $N P \subseteq D T I M E\left(n^{\text {polylog }(n)}\right)$.

The reduction: We create an instance $\bar{G}=(\bar{V}, \bar{E})$ of the DiRECTED PDS problem from a given instance $G=(A, B, E)(\mathcal{H}=(\mathcal{A}, \mathcal{B}, \mathcal{E}))$ of the MinREP problem.

1. Start with a copy of each node in $A \cup B$ in $\bar{G}$. For convenience, we use the same notation for nodes (and sets of nodes) in $G$ and their copies in $\bar{G}$.

2. Add a new node $w^{*}$ to the graph $\bar{G}$, and add a directed edge from $w^{*}$ to each node in $A \cup B$.

3. $\forall i \in\left\{1, \ldots, q_{A}\right\}, j \in\left\{1, \ldots, q_{B}\right\}$ if $A_{i} B_{j}$ is a super edge, then do the following:

(a) Let $E_{i j}$ be the set of edges between $A_{i}$ and $B_{j}$ in $G$, and let $\ell_{i j}$ denote $\left|E_{i j}\right|$. We denote the edges in $E_{i j}$ by $e_{1}, e_{2}, \ldots, e_{k}, \ldots, e_{\ell_{i j}}$. 
(b) Let $D_{i j}$ be the graph on $6 \ell_{i j}+1$ nodes as shown in Figure 4(a), In $D_{i j}$ there are 6 nodes $u_{k}, v_{k}, d_{k}, \alpha_{k}, \beta_{k}, \gamma_{k}$ associated with an edge $e_{k}$ of $E_{i j}$. The part of $D_{i j}$ associated with an edge $e_{k}$ is shown in Figure 4(b) note that all these parts share a common node, called the center node, in $D_{i j}$. Make $\lambda=4$ new copies of the graph $D_{i j}$ ( $\lambda$ can be any constant greater than 3). For each edge $e_{k}=a_{k} b_{k} \in E_{i j}$ and for each of the 4 copies of $D_{i j}$, we add a directed edge from $a_{k}$ to $u_{k}$ and a directed edge from $b_{k}$ to $v_{k}$. In addition to these edges, there are directed edges from $w^{*}$ to some nodes inside $D_{i j}$; these directed edges are denoted by a dashed line in Figure 4(a).

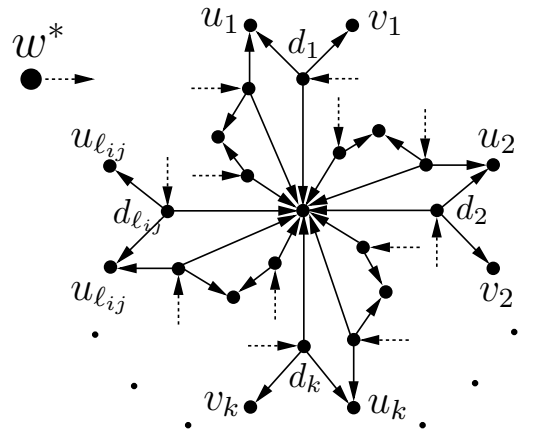

(a) The $D_{i j}$ graph

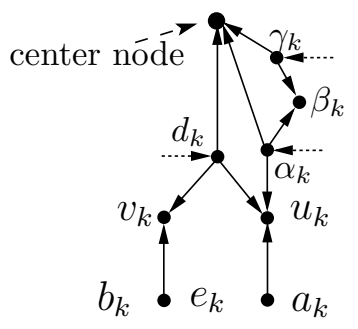

(b) Part of gadget corresponding to an edge $e_{k}$

Figure 4: The cover testing gadget.

4. Let $\bar{G}=(\bar{V}, \bar{E})$ be the obtained graph.

The next lemma shows that the size of an optimal solution in DIRECTED PDS is exactly one more than the size of an optimal solution in the MinReP instance. The number of nodes in the constructed graph is at most $|V(\bar{G})| \leq 1+|V(G)|+7 \lambda|E(G)|$. This will complete the proof of Theorem 4.2 by showing that the above reduction is a gap preserving reduction from MinREP to DiRECTED PDS with the same gap (hardness ratio) as the MinReP problem.

Lemma 4.3 $A^{*} \cup B^{*}$ is an optimal solution to the instance $G=(A, B, E)$ of the MinREP problem if and only if $S^{*}=A^{*} \cup B^{*} \cup\left\{w^{*}\right\} \subseteq V(\bar{G})$ is an optimal solution to the instance $\bar{G}$ of the DIRECTED PDS problem.

Proof: First note that $w^{*}$ should be in any feasible solution; because it has in-degree zero.

Assume that $A^{*} \cup B^{*}$ is a feasible solution for the MinRep instance $G$. We will show that $S=A^{*} \cup B^{*} \cup\left\{w^{*}\right\}$ is a feasible solution to the Directed PDS instance $\bar{G}$. Note that all nodes in $A \cup B$ and some nodes inside the gadgets $D_{i j}$ are power dominated by applying rule (D1) on $w^{*}$. Now, we only need to show that all nodes in the gadgets $D_{i j}$ are power dominated. Consider a super edge $A_{i} B_{j}$ of $\mathcal{H}$. The set $A^{*} \cup B^{*}$ covers all the super edges in $\mathcal{H}$. Hence, there exists a pair of nodes $a_{k} \in A^{*} \cap A_{i}, b_{k} \in B^{*} \cap B_{j}$ that induces an edge of $G$. Since $a_{k}$ and $b_{k}$ are in $S$ their out-neighbors $u_{k}$ and $v_{k}$ in each of the $\lambda=4$ copies of the $D_{i j}$ graph will be power dominated by applying rule (D1). Now node $d_{k}$, that is already power dominated by $w^{*}$, will power dominate the center node by an application of rule (D2). Now we claim that the center node will power dominate the remaining nodes in the gadget $D_{i j}$. Consider the part of gadget (shown in 
Figure 4(b) corresponding to an edge $e_{r} \in E_{i j}$. Note that the nodes $\gamma_{r}, \alpha_{r}$, and $d_{r}$ are already power dominated by $w^{*}$. It is easy to check that the nodes $\beta_{r}, u_{r}, v_{r}$ will be power dominated by sequentially applying rule (D2) on $\gamma_{r}, \alpha_{r}$, and $d_{r}$. This shows that $S$ power dominates all nodes in $\bar{G}$. Therefore, $\operatorname{Opt}(\bar{G})$ is at most $\left|A^{*} \cup B^{*}\right|+1$.

Let $S^{*} \subseteq V(\bar{G})$ be an optimal solution for DiRECTED PDS. As we showed above $w^{*}$ should be in any feasible solution for Directed PDS. Now define $A^{\prime}=A \cap S^{*}$ and $B^{\prime}=B \cap S^{*}$. First we prove that any optimal solution of DiRECTED PDS is contained in $A \cup B \cup\left\{w^{*}\right\}$, and then we show that $A^{\prime} \cup B^{\prime}$ covers all the super edges of $\mathcal{H}$. Suppose that $S^{*}$ contains some nodes not in $A \cup B \cup\left\{w^{*}\right\}$. Hence, there are some gadgets that are not completely power dominated by $S^{*} \cap\left(A \cup B \cup\left\{w^{*}\right\}\right)$. Let $D_{i j}$ be such a gadget. By symmetry each of the $\lambda=4$ copies of $D_{i j}$ is not completely power dominated. Therefore, the optimal solution $S^{*}$ needs to have at least 3 nodes from the 4 copies of $D_{i j}$. By removing these 3 nodes from $S^{*}$ and adding $a_{k} \in A_{i}$ and $b_{k} \in B_{j}$ to $S^{*}$ for some arbitrary edge $e_{k}=a_{k} b_{k} \in E_{i j}$, we can power dominate all 4 copies of $D_{i j}$. This contradicts the optimality of $S^{*}$, and proves that $S^{*} \subseteq A \cup B \cup\left\{w^{*}\right\}$. To see that $A^{\prime} \cup B^{\prime}$ covers all super edges, it is enough to note the following. Suppose no node from any copy of $D_{i j}$ is in the optimal solution, then any $D_{i j}$ can be power dominated only by taking a pair of nodes $a \in A_{i}$ and $b \in B_{j}$ that induces an edge of $G$. Otherwise, any power dominated node in the gadget has at least 2 out-neighbors that are not power dominated, so rule (D2) cannot be applied. This completes the proof of the lemma.

There are several notions for the tree-width of directed graphs such as DAG width [29], directed tree-width [21], and Kelly-width [19. Directed acyclic graphs have width equal to zero for the first two notions [29], and have Kelly-width of 1 . Hence, Theorem 4.2 gives a hardness threshold of $O\left(2^{\log n^{1-\epsilon}}\right)$ even if the directed graph has width $\leq 1$ according to any of the above three notions.

We reformulate DIRECTED PDS in terms of valid colorings of the edges in order to develop an algorithm based on dynamic-programming for DiRECTED PDS. Guo et al. 15] introduced the notion of valid orientations to get a new formulation for PDS (in undirected graphs). They also designed a linear-time dynamic-programming algorithm based on valid orientations for optimally solving PDS on graphs of bounded tree-width. Our method applies to directed graphs such that the underlying undirected graph has bounded tree-width.

Definition 4.4 A coloring of a directed graph $G=(V, E)$ is a partitioning of the edges in $G$ into red and blue edges. We denote a coloring by $\mathcal{C}=\left(V, E_{r} \cup E_{b}\right)$ where $E_{r}$ is the set of red edges and $E_{b}$ is the set of blue edges.

We reformulate the DIRECTED PDS problem via a so-called valid coloring of directed graphs; informally speaking, these colorings "model" the application of rules (D1) and (D2) of DiRECTED PDS.

Definition 4.5 $A$ valid coloring $\mathcal{C}=\left(V, E_{r} \cup E_{b}\right)$ of a directed graph $G=(V, E)$ is a coloring of $G$ with the following properties:

1. No two antiparallel edges can be colored red.

2. The subgraph induced by the red edges, $G_{r}=\left(V, E_{r}\right)$, has the following properties:

(a) $\forall v \in G: d_{G_{r}}^{-}(v) \leq 1$, and

(b) $\forall v \in G: d_{G_{r}}^{-}(v)=1 \Longrightarrow d_{G_{r}}^{+}(v) \leq 1$. 
3. G has no dependency cycle. A dependency cycle is a sequence of directed edges whose underlying undirected graph forms a cycle such that all the red edges are in one direction, all the blue edges are in the other direction, and there are no two consecutive blue edges.

We call a node an origin of $\mathcal{C}$ if it has no incoming edges in $G_{r}$.

Our dynamic-programming algorithm for DiRECTED PDS is based on the following lemma.

Lemma 4.6 Given a directed graph $G$ and $S \subseteq V(G), S$ power dominates $G$ if and only if there is a valid coloring of $G$ with $S$ as the set of origins.

Proof: Suppose $S \subseteq V$ power dominates $G$. Then we give a valid coloring $\mathcal{C}$ with $S$ as the set of origins by coloring the edges in $G$ according to the way that $S$ power dominates $G$. We color an edge $(v, w)$ red if node $w$ is power dominated by applying the power domination rules on $v$; either by the domination rule (D1) or by the propagation rule (D2). Note that when we apply the propagation rule (D2), then we do not power dominate the previously power dominated nodes. Also when we apply rule (D1) on $v$, then we power dominate all (not some subset of) neighbors of $v$ that are not power dominated. We write $v<u$ when a node $u$ is power dominated after $v$. It is easy to check that with this coloring the degree requirements are satisfied; each node can be power dominated only once, and if it is a power dominated node (not in $S$ ), then it cannot power dominate more than one of its out-neighbors due to rule (D2). Now, we need to prove that there is no dependency cycle. By way of contradiction, suppose that $C^{*}=u_{1}, u_{2}, \ldots, u_{m}$ is a dependency cycle. Focus on the edges of $C^{*}$. Call the direction of the red edges forward, and call the direction of the blue edges backward. Observe that a dependency cycle has $\geq 1$ red edges, and each of its red edges corresponds to an application of rule (D2). Assume that all the edges in $C^{*}$ are red. Then the red edges $\left(u_{i}, u_{i+1}\right)$ imply that $u_{i}<u_{i+1}$ for all $i=1,2, \ldots, m-1$; therefore $u_{1}<u_{2}<\cdots<u_{m}$, but this is a contradiction since the last red edge from $u_{m}$ back to $u_{1}$ implies that $u_{m}<u_{1}$. Hence, there is no dependency cycle with all edges colored red. Now, assume that the dependency cycle $C^{*}$ has some blue edges. We show that a similar contradiction occurs when there are no two consecutive blue edges. Consider a blue edge $(u, v)$ of $C^{*}$ and note that the other edge of $C^{*}$ incident to $u$ is a red edge, say $(u, w)$. By rule (D2), we see that $v$ should be power dominated before $u$ can power dominate $w$; thus we have $w>v$. Repeating this argument, we get an ordering for the occurrences of power domination of some of the nodes in $C^{*}$ that gives a contradiction, e.g., if $m$ is even and the edges of $C^{*}$ are alternatively blue and red (starting with blue) we get $u_{1}<u_{3}<u_{5}<\cdots<u_{m-1}<u_{1}$ (see Figure 5 for an example). Hence, $G$ has a valid coloring with $S$ as the set of origins.

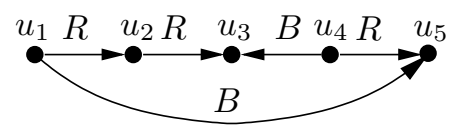

Figure 5: The blue edge $\left(u_{4}, u_{3}\right)$ means that $u_{3}$ should be power dominated before we can power dominate $u_{5}$ by applying rule (D2) on $u_{4}$; thus $u_{3}<u_{5}$. Again the blue edge from $u_{1}$ to $u_{5}$ implies that $u_{5}<u_{2}$. Finally, the red edge $\left(u_{2}, u_{3}\right)$ shows that $u_{2}<u_{3}$. Combining these dependencies we get $u_{3}<u_{5}<u_{2}<u_{3}$. This is a contradiction, and shows that there cannot be a dependency cycle in a coloring obtained from the application of rules (D1) and (D2) of DiRECTED PDS.

Now suppose that $G$ has a valid coloring $\mathcal{C}=\left(V, E_{r} \cup E_{b}\right)$ with $S \subseteq V(G)$ as the set of origins. The nodes in $S$ and all of their out-neighbors in $G_{r}=\left(V, E_{r}\right)$ are power dominated by applying 
the rule (D1). Now we prove that $S$ will power dominate all nodes in $G$. Suppose that this does not happen. Let $X \subset V$ be the maximal set of nodes that can be power dominated by $S$. We claim that there is at least one red edge from $X$ to $V \backslash X$. Note that all of the origins are in $X$, so each of the nodes in $V \backslash X$ has in-degree 1 in $G_{r}$. Hence, if there is no red edge from $X$ to $V \backslash X$, then there should be a directed cycle of red edges in $G[V \backslash X]$. This is not possible since there are no dependency cycles. Therefore there is at least one red edge from $X$ to $V \backslash X$. Let $e_{1}=\left(x_{1}, y_{1}\right), \ldots, e_{k}=\left(x_{k}, y_{k}\right)$ be all of the red edges from $X$ to $V \backslash X$. If some $x_{i}$ has all of its outneighbors in $X$ except $y_{i}$, then by applying rule (D2) on $x_{i}$ the node $y_{i}$ will be power dominated. By the maximality assumption of $X$ this cannot happen. Therefore, each $x_{i}$ has another out-neighbor, say $z_{i}$, in $V \backslash X$. Then $\left(x_{i}, z_{i}\right)$ is a blue edge, otherwise, $x_{i}$ would be an origin and $y_{i}$ would be power dominated by applying rule (D1) on $x_{i}$. Now, we construct a dependency cycle as follows: starting from $x_{1}$, use a blue edge to move to a node $z_{1}$ in $V \backslash X$; then move in the reverse direction over a sequence of red edges $\left(z_{2}, z_{1}\right),\left(z_{3}, z_{2}\right), \cdots$ until we reach a red edge $\left(z_{i}, z_{i-1}\right)$ with $z_{i-1} \in V \backslash X$ and $z_{i} \in X$ (such an edge exists since $G[V \backslash X]$ has no directed cycle of red edges); note that $\left(z_{i}, z_{i-1}\right)$ is one of the red edges $\left(x_{1}, y_{1}\right), \cdots,\left(x_{k}, y_{k}\right)$. If $z_{i}=x_{1}$, then we have a dependency cycle; otherwise, we again use a blue edge $\left(z_{i}, z_{i+1}\right)$ to move to a node in $V \backslash X$. By repeating these steps, we will eventually find a dependency cycle. Note that all the blue edges are in one direction, and all the red edges are in the other direction. This is a contradiction, since $\mathcal{C}$ has no dependency cycle. Hence, we have $X=V$, so $S$ power dominates $G$.

Theorem 4.7 Given a directed graph $G$ and a tree decomposition of width $k$ of its underlying undirected graph, DIRECTED PDS can be optimally solved in $O\left(c^{k^{2}} \cdot n\right)$ time for a global constant $c$.

A consequence of the above theorem is a linear-time algorithm for solving the DIRECTED PDS problem optimally on directed graphs, given a bounded tree-width decomposition of the underlying undirected graph. Also since the tree-width decomposition for graphs with bounded tree-width can be computed in polynomial-time [5], there is a polynomial-time algorithm to solve DiRECTED PDS optimally on the class of directed graphs such that the underlying undirected graph has bounded tree-width.

\section{Conclusions}

We studied the PDS problem from the perspective of approximation algorithms. We introduced a natural extension of the problem to directed graphs. We showed that both problems have a threshold of $O\left(2^{\log n^{1-\epsilon}}\right)$ for the hardness of approximation. We presented an $O(\sqrt{n})$ approximation algorithm for PLANAR PDS. We designed a dynamic-programming algorithm for solving the DiRECTED PDS problem optimally in linear-time for those directed graphs whose underlying undirected graph has bounded tree-width.

Here, we describe an algorithm with an approximation guarantee of $O\left(\frac{n}{\log n}\right)$ for the PDS problem. The algorithm works as follows. Partition the nodes of the graph $G$ into $\log n$ equal-sized sets $V_{1}, V_{2}, \cdots$. Next, consider all possible ways of picking these sets (we pick all nodes in a set). Among all these different candidates, output the one that power dominates $G$ and has the minimum number of nodes. Note that in the algorithm we only consider $2^{\log n}=n$ different candidates. Clearly, the algorithm runs in polynomial time, since the feasibility of each candidate can be tested in polynomial time. Let $S^{*}$ be an optimal solution. It is easy to see that the set of $V_{i}$ 's that 
intersect $S^{*}$ forms a feasible solution for the PDS problem in $G$; this solution has size at most $\frac{n}{\log n} \cdot\left|S^{*}\right|$. This establishes the approximation guarantee. The same algorithm and analysis applies to the DiRECTED PDS problem.

Proposition 5.1 There is a polynomial time $\frac{n}{\log n}$-approximation algorithm for both the PDS problem and the DIRECTED PDS problem.

There is a gap between our hardness threshold of $O\left(2^{\log n^{1-\epsilon}}\right)$ and our approximation guarantee of $O\left(\frac{n}{\log n}\right)$, and narrowing this gap is an open question.

A major open question in the area is whether there exists a PTAS (polynomial time approximation scheme) for PLANAR PDS. A first step may be to obtain an improvement on our approximation guarantee of $O(\sqrt{n})$. There has been a lot of research on designing PTASs for NP-hard problems on planar graphs. Some of the most important developments are the outerplanar layering technique by Baker [3], and the bidimensionality theory by Demaine and Hajiaghayi [10]. Unfortunately, these methods do not apply to PLANAR PDS.

Baker [3] showed that the Dominating SET problem in planar graphs has a PTAS. In the Baker method we first partition the graph into smaller graphs. Then we solve the problem optimally on each subgraph, and finally we return the union of the solutions as a solution for the original graph. The example in Figure 1 shows that this method does not apply to PLANAR PDS. The size of an optimal solution is 1 , but if we apply the Baker method, then the size of the output solution will be at least as large as the number of subgraphs in the partition which can be $\Theta(n)$.

Demaine and Hajiaghayi [10] introduced the bidimensionality theory and used it to obtain PTASs for several variants of the Dominating SET problem on planar graphs. An important property of bidimensionality is that when an edge is contracted the size of an optimal solution should not increase. Consider the example in Figure 6. If we contract edges $e_{1}, e_{2}, \ldots, e_{n}$ in $G$, then we get the graph $G^{\prime}$. It can be checked that $\operatorname{Opt}(G)=1$ but $\operatorname{Opt}\left(G^{\prime}\right)=\Theta(n)$. Thus the bidimensionality theory does not apply to PLANAR PDS, since the optimum value may increase when an edge is contracted.

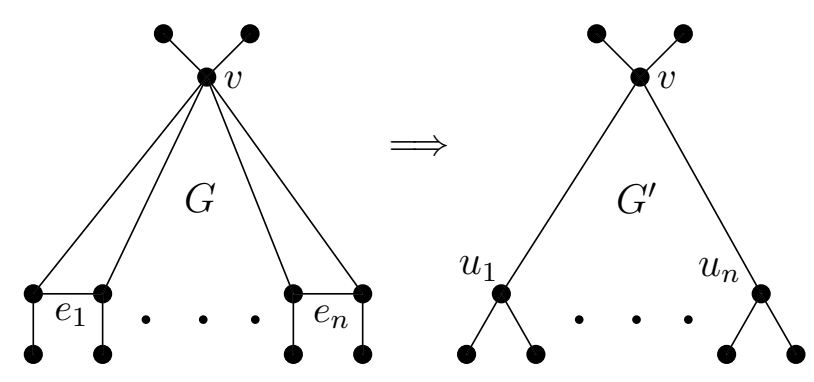

Figure 6: Optimal value of PDS increases when edges are contracted.

Lastly, we consider some variations of greedy algorithms for PDS and show that they perform very poorly even on planar graphs. In contrast, for other related problems such as Dominating SET and Set Covering, greedy algorithms perform well since they achieve a logarithmic approximation guarantee, and no substantial improvement is possible by any polynomial time algorithm, under complexity assumptions like $\mathrm{P} \neq \mathrm{NP}$. The most natural greedy algorithm for PDS is the one that starts with $S=\emptyset$, and in each step, adds a new node $v$ to the current solution $S$ such that $v$ power dominates the maximum number of new nodes. 
Unfortunately, this greedy algorithm may find a solution $S$ such that $|S| \geq \Theta(n) \cdot \operatorname{Opt}(G)$. To see this, consider a graph $G$ that is obtained from a $9 \ell \times 9 m$ grid by subdividing all row-edges, except with minor changes in the four corners as shown in Figure $7(\mathrm{a})$. Partition the graph $G$ into $9 \times 9$ grids (ignoring the nodes introduced by subdivision), see Figure 7(b), It is easy to check that any single node can power dominate at most 7 nodes, and the center node of any one of the $9 \times 9$ grids achieves this maximum. So the greedy algorithm at the first iteration may pick the center node of any one of the $9 \times 9$ grids. Assuming all nodes picked by the algorithm so far have been these center nodes, we see that picking another center node maximizes $\left|\mathcal{P}_{S \cup\{v\}} \backslash \mathcal{P}_{S}\right|$ over all $v \in V$. So the greedy algorithm could continue picking center nodes, and after that possibly picking other nodes until it finds a feasible solution $S$. The size of the output $S$ is at least $m \cdot \ell=\Theta(n)$. By

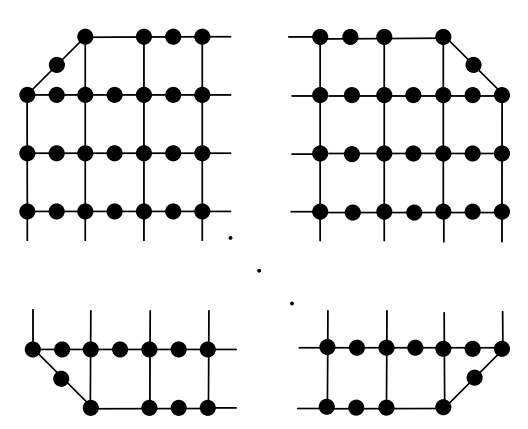

(a) Grid

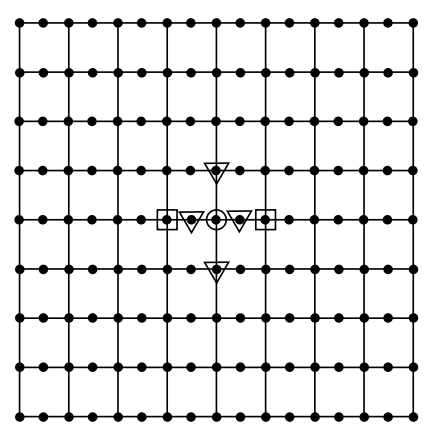

(b) $9 \times 9$ grid

Figure 7: Bad example for the greedy algorithm

Proposition 3.12, we have $\operatorname{Opt}(G)=\Theta(\ell)$. Now by fixing $\ell=\Theta(1)$ we can see that the size of the output solution can be bigger than $\operatorname{Opt}(G)$ by a factor of $\Theta(n)$.

Proposition 5.2 The greedy algorithm for the PDS problem may find a solution $S$ such that $|S| \geq$ $\Theta(n) \cdot \operatorname{Opt}(G)$.

We will consider two other variations of the greedy algorithm, namely Proximity and Cleanup. We have examples of PLANAR PDS showing that these variations of the greedy algorithm perform poorly.

Proximity: In each step of the Proximity algorithm we choose a node such that the set of all power dominated nodes induces a connected subgraph, and subject to this, the number of newly power dominated nodes is maximized. Informally, this is to escalate the use of the propagation rule.

The bad example for the proximity version of the greedy algorithm is obtained by modifying the center row of the $h \times(2 m+1)$ grid, as shown in Figure 8 , by inserting $\ell$ subdividing nodes into the edges in the middle row, and also subdividing all of the other row-edges except some of the corner edges. Figure 8 illustrates an example of such a grid for $l=5$ and $h=9$ rows, but for a bad example for the proximity greedy algorithm we need $h$ to be sufficiently large constant $(h=17$ suffices). We use the figure for illustration, to show the working of the proximity greedy algorithm.

It is easy to check that by picking all nodes of the first column we can power dominate the entire graph, so the optimal solution is $\Theta(1)$. The proximity greedy algorithm starts by picking a node that power dominates maximum number of nodes (which is $17=2 \ell+7$ ); any white node satisfies this requirement. Therefore the algorithm may pick for example the first white node (from the 
left). It is easy to check that in the next step the algorithm will pick the white node to the right of the first one, since all of the power dominated region stays connected and also it power dominates maximum number of new nodes (which is $16=2 \ell+6$ ). The algorithm continues picking all white nodes and at the end it will pick possibly more nodes to get a feasible solution. (The shaded region shown in Figure 8 indicates the nodes that will be power dominated by picking all white nodes.) Therefore, the size of the solution found by the algorithm is at least $m=\Theta(n)$. Hence, the proximity greedy algorithm may find a feasible solution that is $\Theta(n)$ times worse than the optimal solution.

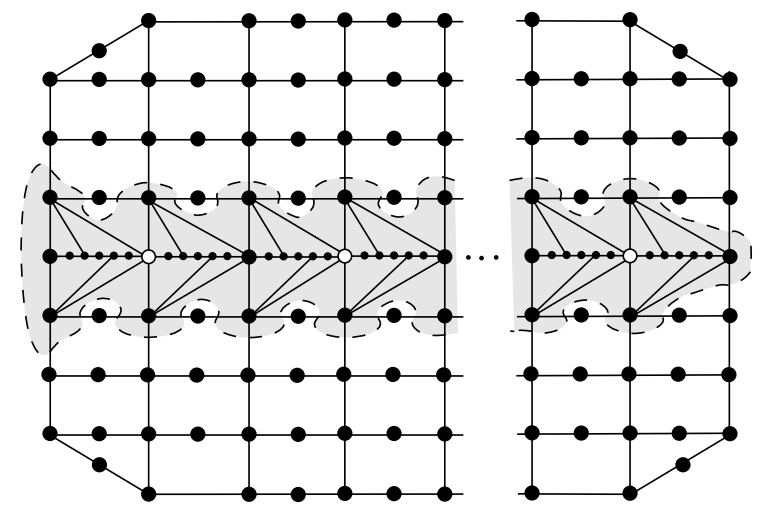

Figure 8: Bad example for the proximity greedy algorithm

Cleanup Step: Some of the recent approximation algorithms, especially some based on the primaldual method, use a clean up step at the end: this step removes redundant elements from the solution in some sequential order. In the Cleanup algorithm, we first run the greedy algorithm to find a solution (node set) $S$, then we repeatedly remove nodes from $S$, until $S$ is an inclusionwise minimal power dominating set. Although a cleanup step may substantially improve on the solution found by the greedy algorithm on some examples, this does not hold for all examples.

The same bad example for the proximity version is also a bad example for the cleanup version of the greedy algorithm. The cleanup greedy algorithm may again pick the first white node (from the left), and after picking this white node, it may pick the third white node. Since both of them power dominate maximum number of new nodes (which is $17=2 \ell+7$ ). Note that in the original greedy algorithm there is no need to have a connected subgraph induced on power dominated nodes. The algorithm continues to pick all the odd indexed white nodes, and after that it will start picking the even indexed white nodes since any one of them power dominates maximum number of new nodes (which is at least $15=2 \ell+5$ ). At this stage of the algorithm the set of power dominated nodes are those in the shaded region of Figure 8. It is easy to check that we need to pick nodes from both upper and lower parts in order to power dominate the entire graph. The greedy algorithm may pick some nodes from the leftmost column in the top part and some nodes from the rightmost column in the bottom part to power dominate the entire graph. Now we start doing the cleanup process. It can be checked that if we remove any two consecutive white nodes from the obtained solution, the graph cannot be power dominated completely. So we need to keep at least half of the white nodes. Therefore, the size of the output solution at the end of cleanup process is at least $\frac{m}{2}=\Theta(n)$, but the optimal solution is just $\Theta(1)$ as before. 


\section{References}

[1] A. Aazami and M. D. Stilp. Approximation algorithms and hardness for domination with propagation. In Proceedings of the 10th International Workshop on Approximation Algorithms for Combinatorial Optimization Problems, volume 4627 of LNCS, pages 1-15. Springer, 2007.

[2] J. Alber, H. L. Bodlaender, H. Fernau, T. Kloks, and R. Niedermeier. Fixed parameter algorithms for dominating set and related problems on planar graphs. Algorithmica, 33(4):461493, 2002.

[3] B. S. Baker. Approximation algorithms for NP-complete problems on planar graphs. J. ACM, 41(1):153-180, 1994.

[4] T. L. Baldwin, L. Mili, M. B. Boisen, and R. Adapa. Power system observability with minimal phasor measurement placement. IEEE Transactions on Power Systems, 8(2):707-715, 1993.

[5] H. L. Bodlaender. A linear-time algorithm for finding tree-decompositions of small treewidth. SIAM J. Comput., 25(6):1305-1317, 1996.

[6] D. J. Brueni. Minimal PMU placement for graph observability, a decomposition approach. Master's thesis, Virginia Polytechnic Institute and State University, Blacksburg, VA, 1993.

[7] D. J. Brueni and L. S. Heath. The PMU placement problem. SIAM J. Discret. Math., 19(3):744-761, 2005.

[8] V. Chvatal. A greedy heuristic for the set covering problem. Math. Oper. Res., 4:233-235, 1979.

[9] B. Courcelle, J. A. Makowsky, and U. Rotics. Linear time solvable optimization problems on graphs of bounded clique width. In Proceedings of the 24th International Workshop on Graph-Theoretic Concepts in Computer Science, volume 1517 of LNCS, pages 1-16. Springer, 1998.

[10] E. D. Demaine and M. T. Hajiaghayi. Bidimensionality: new connections between FPT algorithms and PTASs. In Proceedings of the 16th Annual ACM-SIAM Symposium on Discrete Algorithms, pages 590-601, 2005.

[11] R. Diestel. Graph Theory. Springer-Verlag, New York, 2nd edition, 2000.

[12] M. Dorfling and M. A. Henning. A note on power domination in grid graphs. Discrete Applied Mathematics, 154(6):1023-1027, 2006.

[13] U. Feige. A threshold of $\ln n$ for approximating set cover. J. ACM, 45(4):634-652, 1998.

[14] M. R. Garey and D. S. Johnson. Computers and Intractability: A Guide to the Theory of NP-Completeness. W. H. Freeman and Co., New York, NY, USA, 1979.

[15] J. Guo, R. Niedermeier, and D. Raible. Improved algorithms and complexity results for power domination in graphs. In Proceedings of the 15th International Symposium on Fundamentals of Computation Theory, volume 3623 of LNCS, pages 172-184. Springer, 2005 (to appear in Algorithmica). 
[16] T. W. Haynes, S. M. Hedetniemi, S. T. Hedetniemi, and M. A. Henning. Domination in graphs applied to electric power networks. SIAM J. Discrete Math., 15(4):519-529, 2002.

[17] T. W. Haynes, S. T. Hedetniemi, and P. J. Slater. Domination in Graphs: Advanced Topics. Marcel Dekker, New York, 1998.

[18] T. W. Haynes, S. T. Hedetniemi, and P. J. Slater. Fundamentals of Domination in Graphs. Marcel Dekker, New York, 1998.

[19] P. Hunter and S. Kreutzer. Digraph measures: Kelly decompositions, games, and orderings. In Proceedings of the 18th Annual ACM Symposium on Discrete Algorithms, pages 637-644, Philadelphia, PA, USA, 2007.

[20] D. S. Johnson. Approximation algorithms for combinatorial problems. J. Comput. Syst. Sci., 9(3):256-278, 1974.

[21] T. Johnson, N. Robertson, P. D. Seymour, and R. Thomas. Directed tree-width. J. Comb. Theory, Ser. B, 82(1):138-154, 2001.

[22] T. Kloks. Treewidth, Computations and Approximations, volume 842 of LNCS. Springer, 1994.

[23] J. Kneis, D. Mölle, S. Richter, and P. Rossmanith. Parameterized power domination complexity. Inf. Process. Lett., 98(4):145-149, 2006.

[24] G. Kortsarz. On the hardness of approximating spanners. Algorithmica, 30(3):432-450, 2001.

[25] C. S. Liao and D. T. Lee. Power domination problem in graphs. In Proceedings of the 11th International Computing and Combinatorics Conference, volume 3595 of LNCS, pages 818828. Springer, 2005.

[26] L. Lovász. On the ratio of optimal integral and fractional covers. Discrete Mathematics, 13:383-390, 1975.

[27] C. Lund and M. Yannakakis. On the hardness of approximating minimization problems. $J$. ACM, 41(5):960-981, 1994.

[28] L. Mili, T.L. Baldwin, and A.G. Phadke. Phasor measurements for voltage and transient stability monitoring and control. In Proceedings of the EPRI-NSF Workshop on Application of Advanced Mathematics to Power Systems, 1991.

[29] J. Obdrzálek. Dag-width: connectivity measure for directed graphs. In Proceedings of the 17th Annual ACM Symposium on Discrete Algorithms, pages 814-821. ACM Press, 2006.

[30] P. Slavík. A tight analysis of the greedy algorithm for set cover. In Proceedings of the 28th Annual ACM Symposium on Theory of Computing, pages 435-441, New York, NY, USA, 1996. ACM Press.

[31] M. D. Stilp. On power dominating sets. Master's thesis, Combinatorics and Optimization, University of Waterloo, Ontario, Canada, 2006. 


\section{A Dynamic Programming}

In this section we describe our dynamic-programming algorithm for the DIRECTED PDS problem. This algorithm is similar to the dynamic-programming algorithm designed by Guo et al. [15] to optimally solve PDS for undirected graphs with bounded tree-width. It is known that any tree decomposition of width- $k$ can be transformed to a nice tree decomposition with width $k$ in lineartime [22] (Lemma 13.1.3). So we can assume that we are given a nice tree decomposition of the underlying undirected graph of $G$ call it $\left\langle\left\{X_{i} \mid i \in I\right\}, T\right\rangle$. Let $T_{i}$ denote the subtree of $T$ rooted at $T$-node $i$, and $Y_{i}=\left(\bigcup_{j \in V\left(T_{i}\right)} X_{j}\right) \backslash X_{i}$. Also let $G_{i}$ be the subgraph induced by $Y_{i} \cup X_{i}$, i.e. $G_{i}=G\left[Y_{i} \cup X_{i}\right]$, and let $G_{i}^{\prime}=G\left[X_{i}\right]$.

Consider a valid coloring $\mathcal{C}$ of the graph $G$. We store the color of the edges in each bag by assigning a state to that bag (the formal definition of a state will follow). We can reconstruct the coloring $\mathcal{C}$ from the states of all bags in the tree decomposition of $G$; so there is no need to store the coloring $\mathcal{C}$ in the dynamic-programming.

The state of a bag: Given a coloring $\mathcal{C}$, the state of a bag $X_{i}$ describes the coloring of the edges in $G_{i}^{\prime}$. In order to detect the dependency cycles in the coloring $\mathcal{C}$ without reconstructing the whole coloring, we need to store some more information in a state. This extra information enables us to detect a dependency cycle in $G_{i}$ which goes through $X_{i}$, by considering only the state of the bag $X_{i}$. A bag state $s$ contains the following: state of each edge, state of each node, and state of each pair of nodes in $G_{i}^{\prime}=G\left[X_{i}\right]$.

- State of an edge: The state of an edge $e \in E\left(G\left[X_{i}\right]\right)$ denoted by $s(e)$ is the color that is assigned to $e$ in the coloring $\mathcal{C} ; s(e) \in\{R, B\}$.

- State of a node: The state of a node $v \in X_{i}$ denoted by $s(v)$ shows the number of red edges between $v$ and $Y_{i}$.

$-s(v)=1:$ There is exactly one red edge from a node in $Y_{i}$ to $v$ and no red edge from $v$ to $Y_{i}$,

$-s(v)=2$ : There is exactly one red edge from a node in $Y_{i}$ to $v$ and exactly one red edge from $v$ to $Y_{i}$,

$-s(v)=3$ : There is no red edge between $Y_{i}$ and $v$,

$-s(v)=4$ : There are at least two red edges from $v$ to $Y_{i}$ and no red edge from $Y_{i}$ to $v$,

$-s(v)=5$ : There is exactly one red edge from $v$ to $Y_{i}$ and no red edge from $Y_{i}$ to $v$.

- State of a pair of nodes: A dependency path from $u$ to $v$ is a path $P$ where all red edges in $P$ are directed from $u$ to $v$ and all blue edges are directed from $v$ to $u$. We categorize dependency paths according to the color of their first and last edges. There are 4 possible types $R R, R B, B R, B B$; for example a path of type $R B$ is a path with the first edge colored red and the last edge colored blue. For a pair $(u, v) \in X_{i} \times X_{i}(u \neq v)$ the state of $(u, v)$ denoted by $s(u, v)$ shows the type of dependency paths from $u$ to $v$ in $G\left[Y_{i} \cup\{u, v\}\right]$; that is, $s(u, v) \subseteq\{R R, R B, B R, B B\}$. Note that there are $2^{4}=16$ different states for each pair of nodes.

Detecting dependency cycles: An important part of the dynamic-programming algorithm is to detect dependency cycles in the coloring $\mathcal{C}$. Assume we are at bag $X_{i}$ and we are given the bag state $s$ corresponding to the coloring $\mathcal{C}$. We can detect the dependency cycles in $G_{i}^{\prime}=G\left[X_{i}\right]$ by 
enumerating all possible cycles; note that the coloring of edges in $G_{i}^{\prime}$ is given in the state $s$. The dependency cycles in $G_{i}$ can be detected by considering the state of each pair of nodes in $X_{i}$. For example assume that $R B \in s(u, v)$ and $R R \in s(v, u)$. Then, by combining a dependency path of type $R B$ from $u$ to $v$ and a dependency path of type $R R$ from $v$ to $u$ we obtain a dependency cycle going through $u$ and $v$ in $G_{i}$.

Let us denote by $\Lambda_{i}$ the set of all possible states for the bag $X_{i}$. The dynamic-programming will compute a mapping $A_{i}: \Lambda_{i} \rightarrow \mathbb{N} \cup\{+\infty\}$. For a bag state $s \in \Lambda_{i}$ the value $A_{i}(s)$ is the minimum number of origins in an optimal valid coloring $\mathcal{C}$ of $G_{i}$ under the restriction that the state of nodes, edges, and pairs of nodes in $X_{i}$ is given by $s$. Now, we describe how our dynamic-programming works.

Step 1: (Initialization): In this step for each leaf node $i$ of $T$, we initialize the mapping $A_{i}$ as follows. For a given state $s$, we define $A_{i}(s)$ as $+\infty$ if $s$ has a dependency cycle, a node $v$ with $s(v) \neq 3$, or a pair of nodes $u$ and $v$ such that $s(u, v) \neq \emptyset$. Otherwise, we define $A_{i}(s)$ as the number of nodes with no in-coming red edges in the coloring defined by $s$.

Step 2: (Bottom-Up Computation): After initialization, we visit the nodes in $T$ in a bottomup fashion and at each bag $X_{i}$ we compute the mapping $A_{i}$ corresponding to $X_{i}$. The update process depends on the type of $T$-nodes that we are considering. Here, we only consider the update process at an InSERT NoDE. The other cases are similar to this one.

Insert Node: Suppose $i$ is an insert node with the child $j$, and assume that $X_{i}=X_{j} \cup\{x\}$. For each bag state $s \in \Lambda_{i}$ do the following:

1. Check whether the coloring given by $s$ forms a valid coloring of $G_{i}^{\prime}$; if not, define $A_{i}(s)=+\infty$.

2. Compute the set $\Lambda_{j}(s)$ containing bag states of $j$ that are "compatible" with the bag state $s$.

3. For each $s^{\prime} \in \Lambda_{j}(s)$, check if a valid coloring of $G_{j}$ "compatible" with $s^{\prime}$ can be extended to a valid coloring of $G_{i}$ "compatible" with $s$.

4. Compute $A_{i}$ based on the mapping $A_{j}$.

Compatible bag state (Step 2): A bag state $s^{\prime} \in \Lambda_{j}$ is said to be compatible with the bag state $s \in \Lambda_{i}$ if the state of each node, each edge, and each pair of nodes in $V\left(G_{j}^{\prime}\right)$ in the bag state $s^{\prime}$ is the same as the corresponding state in the bag state $s$. If $s(x) \neq 3$, or $\exists v \in X_{j}: s(x, v) \neq \emptyset \vee s(v, x) \neq \emptyset$ then we define $\Lambda_{j}(s)=\emptyset$.

Detecting dependency cycles (Step 3): The conditions of a valid coloring can be violated due to degree constraints on the new node $x$, or by the existence of a dependency cycle going through $x$. Both these cases can be tested by considering the bag states $s$ and $s^{\prime}$.

Computing $A_{i}$ (Step 4): The addition of $x$ may change the number of origins in $G_{i}$. The node $x$ will be an origin if it has at least one outgoing red edge in $s$. But an origin node $v \in X_{j}$ (in $s^{\prime}$ ) that has an incoming red edge from $x$, is no longer an origin. So by considering the red edges going out of $x$ we can update the number of origins and compute the mapping $A_{i}$. If the coloring compatible with $s$ is not a valid coloring, then we define $A_{i}(s)$ to be $+\infty$.

Step 3: (At root $r$ ): Finally, we compute the number of origins in an optimal valid coloring of $G$ by finding the minimum of $A_{r}(s)$ over all possible $s \in \Lambda_{r}$. It is easy to see that each bag $X_{i}$ has at most $16^{(k+1)^{2}} \cdot 5^{k+1} \cdot 2^{(k+1)^{2}}$ states; note that $\left|X_{i}\right| \leq(k+1)$. It can be checked that the total running time of our algorithm is $O\left(c^{k^{2}} \cdot n\right)$, for some global constant $c$. 